\title{
Early Onset Colorectal Cancer in the United Arab Emirates, Where do we Stand?
}

\author{
Humaid 0. 'Al-Shamsi 1-4*, Adhari Abdullah Alzaabi ${ }^{\text {* }}$, Amr Hassan ${ }^{1,2,4}$, \\ Ibrahim Abu-Gheida ${ }^{1,2,4}$ and Sadir Alrawi ${ }^{1,2,4}$ \\ ${ }^{1}$ Department of Oncology, Burjeel Cancer Institute, Burjel Medical City, Abu Dhabi, \\ United Arab Emirates \\ ${ }^{2}$ Innovation and Research Center, Burjeel Cancer Institute, Burjel Medical City, Abu \\ Dhabi, United Arab Emirates \\ ${ }^{3}$ College of Medicine, University of Sharjah, Sharjah, United Arab Emirates \\ ${ }^{4}$ Emirates Oncology Society, Dubai, United Arab Emirates \\ ${ }^{5}$ Sultan Qaboos University, Sultanate of Oman \\ *Corresponding Author: Humaid O. Al-Shamsi, Associate Professor, University of \\ Sharjah, Sharjah, United Arab Emirates.
}

Received: October 23, 2020

Published: October 28, 2020

(C) All rights are reserved by Humaid 0.

Al-Shamsi., et al.

\begin{abstract}
The burden of CRC among young adults has been recognized recently as an area that needs further research. Early onset colorectal cancer (EOCRC) is defined as a colorectal cancer that is diagnosed $<50$ years of age. there is no consensus between studies if EOCRC is similar or if it has distinct molecular, clinical and epidemiologic features compared to adult onset CRC. EOCRC is usually characterized by an advanced stage at presentation, frequent high-grade cancers and poor prognosis. In the UAE there is anecdotal evidence that > $41 \%$ of UAE colon cancer patients are people younger than 50 years, and $>22 \%$ of the colon cancer cases are patients below the age of 40. Initiating screening earlier, at age 45 years, is a "qualified" recommendation from the American Cancer Society, yet there is no international recommendations to start earlier than 45 years of age. In the UAE the screening age has been set to start at the age of 40 , largely due to the earlier data that shows that $>41 \%$ of UAE colon cancer patients are people younger than 50 years. Currently there are no published data regarding the outcome of early CRC screening in the UAE. Targeted surveillance, prevention, and treatment are needed to reduce the cancer burden in this underserved age group.
\end{abstract}

Keywords: COVID-19; Oncology; Asymptomatic; Infection Prevention and Control

\section{Introduction}

An increased incidence of cancers in younger individuals $<50$ years has been observed for a variety of cancers worldwide [1]. This raises important questions of the best time to start screening individuals for cancer, whether there are different biomarkers that should be used, and whether these earlier cancers have different mutations and perhaps require different therapies compared to tumors in older patients [2]. As an example, UAE women are being diagnosed 10 years earlier than in the western countries, with disease with worse prognosis compared with other breast cancer subtypes [3-5] (See also figure 1A).

\section{Early onset colon cancer}

Colorectal cancer (CRC) is considered the third most common cancer and leading cause of death worldwide in both genders [7]. Despite the slowly and steadily decreasing of both the incidence and mortality of CRC in western countries, the incidence of early-onset CRC has nearly doubled since the 1990s [8]. Early onset colorectal cancer (EOCRC) is defined as a colorectal cancer that is diagnosed before the screening age, i.e. $<50$ years of age. According to the American Cancer Society, between 1994 and 2016, there was a noticeable decrease in incidence of adult onset CRC ( $>50$ years) 
by $2 \%$ per year. At the same time, they noticed an increase of early onset CRC by $2 \%$ per year [7]. The overall reduction has been mainly attributed to the higher uptake of screening tests, that resulted in early detection and excision of premalignant lesions, and to the increase in risk factor awareness among the population [9]. On the other hand, the increase in EOCRC incidence has not been fully understood till today. In fact, there is a prediction that by 2030 in the USA, $10 \%$ of all colon and $22 \%$ of all rectal cancers are expected to be diagnosed in patients $<50$ years old [10].

\section{Are we dealing with a new disease?}

Interestingly, there is no consensus between studies if EOCRC is similar or if it has distinct molecular, clinical and epidemiologic features compared to adult onset CRC $[11,12]$.

Clinical data showed that compared to adult onset CRC, EOCRC is usually characterized by an advanced stage at presentation, frequent high-grade cancers and poor prognosis [10,13]. Generally, this could be likely explained by the fact that patients at this age are diagnosed due to symptomatic disease or high-risk group screening compared to those older than 50 who are diagnosed based on screening tests mainly. Keeping in mind the fact that many CRCs are asymptomatic, the increase in incidence of EOCRC does not represent a shift in age at diagnosis attributable to earlier detection. Instead, it is likely indicates a real rise in occurrence of CRC in this age group that is not screened at an earlier stages and stay clinically undetected till it becomes symptomatic and advanced [14-16].

\section{CRC in United Arab Emirates}

In UAE, CRC is the second most common cancer among both genders and the most common cancer among males and the third most common cancer in females, The latest data indicates that the highest frequencies of colorectal cases were found among age groups 55 - 59 years (15.2\%), followed by age group 60 - 64 year (11.4\%) (UAE cancer registry 2015). At diagnosis, the median age of both males and females patients with CRC is 51 years in UAE [17] compared to 68 and 72 years in men and women, respectively in USA. For the UAE there is anecdotal evidence that $>41 \%$ of UAE colon cancer patients are people younger than 50 years, and $>22 \%$ of the colon cancer cases are patients below the age of 40 [18]. In a cohort of 6078 patients underwent colonoscopies for diagnostic work up for multiple indications showed 58 cancers detected where $40 \%$ below age $50,22 \%$ below age 40 [17]. In the period from January to December of 2015, a total number of 387 new cases were diagnosed with colorectal cancer in UAE among both UAE citizens and Non-UAE citizens; of which 373 (96.4\%) were malignant colorectal cases and 14 (3.6\%) were carcinoma in situ (UAE cancer registry 2015). Our own analysis of data from the UAE-National Cancer Registry for 2015 shows that in the UAE national population $23.4 \%$, and in the non-UAE national population $41.9 \%$ of colorectal cancer cases occurred before the age of 50 . This compares to just $5.8 \%$ before the age of 50 in a Western (US) population (See figure 1B). There appears to be a separate group of early onset colon cancer, although the number of cases is too small make any firm conclusions.
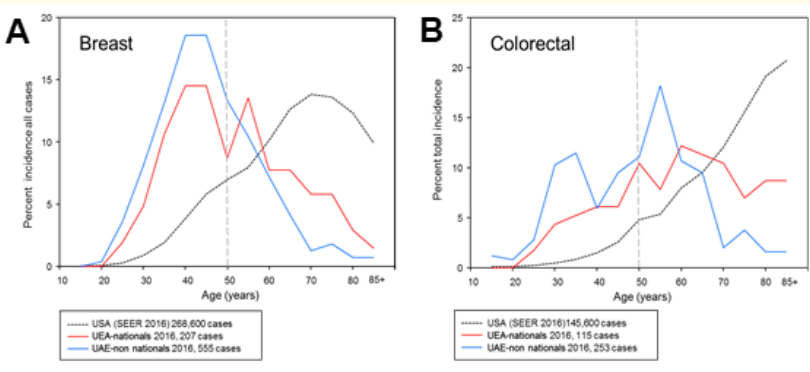

Figure 1: Incidence of breast and colorectal in the UAE 2015. A, for UAE nationals and non-UAE nationals with breast cancer; and $\mathrm{B}$, with colorectal cancer. The data is presented as the percent by age of total reported cases. Also shown for reference is SEER data for the USA 2016 [6].

\section{Screening for EOCRC}

At present, current guidelines do not recommend screening for asymptomatic individuals under the age of 50 unless they have a positive family history or a predisposing inherited syndrome [19]. Initiating screening earlier, at age 45 years, is a "qualified" recommendation from the American Cancer Society (ACS) [20]; this accompanies its strong recommendation to screen at age 50 or older. Data to support starting at age 45 years are limited; the rationale is supported from some but not all modeling analyses [21].

It has been strongly believed that CRC at a younger age is largely associated with hereditary factors. Surprisingly, recent evidence does not support this belief. Genetic risk assessment of a cohort of young CRC patients revealed that only 1 in 5 of these patients do carry a germline genetic mutation and about $25 \%$ of them had a first-degree relative with CRC. The remaining majority of patients did not have any hereditary syndromes and were sporadic with negative family history $[22,23]$. These findings do not underscore 
the hereditary element in EOCRC but it highlights the need for intensive research to decipher the risk factors i.e. biological and environmental that are attributed to this global increase in incidence. Once risk factors associated with EOCRC are identified, they can be incorporated into existing preventive strategies aiming for better prevention and early detection for this global issue.

It is interesting to know that, in the UAE, the screening age has been set to start at the age of 40 , largely due to the earlier data that shows that $>41 \%$ of UAE colon cancer patients are people younger than 50 years [24]. Currently there are no published data regarding the outcome of this early CRC screening in the UAE, it will be interesting to assess the efficacy of such an early intervention for morbidity and mortality outcome. It is paramount to mention that according to non-published data that we reviewed as part of the UAE national cancer mortality reduction committee, only $1.6 \%$ of eligible targeted population had a FIT test or colonscopy in 2017. With all the caveats regarding the accuracy of the data due to numerous factors, our clinical judgment is also in keeping in that, there is a very limited uptake of the CRC screening in the UAE at best. Factors contributing to low uptake of the CRC screening includes a poor level of knowledge on risk factors of CRC in both public and also health care providers, new policies need to focus more on increasing community awareness on cancer preventive measures in UAE [25]. The issue for low CRC screening uptake in UAE population must be considered in any planning for further research to assess screening for EOCRC. Potential methods include non invasive screening methods like fecal immunochemical test [FIT], guaiacbased fecal occult blood test or Epi proColon 2.0@ which detects Septin 9, which is a plasma assay that detects circulating methylated septin 9 DNA, which is hypermethylated in CRC but not in normal colon tissue. The US Food and Drug Administration (FDA) approved an assay for septin-9 DNA (Epi proColon 2.0 (C) as an aid for CRC detection in average-risk patients who refuse screening by any more sensitive guideline-recommended method; however, its sensitivity is considered inadequate as a primary screening strategy. These methods can improve the CRC detection and can be considered for screening of EOCRC in a clinical trials settings to assess efficacy and cost implications.

\section{Conclusion}

In summary, Epidemiological evidence indicates that EOCRC is a current global public health issue that warrants further research. Targeted surveillance, prevention, and treatment are needed to reduce the cancer burden in this underserved age group.

\section{Funding}

Khalifa Bin Zayed Al Nahyan Foundation, Abu Dhabi, United Arab Emirates. Khalifa Bin Zayed Al Nahyan Foundation had no role in the design and conduct of the study; review, or approval of the manuscript; and decision to submit the manuscript for publication. Khalifa Bin Zayed Al Nahyan Foundation provided funding for open-access publication.

\section{Conflict of Interests}

H.O.A received publication funding from Roche Pharmaceuticals Middle East FZCO and Khalifa Bin Zayed Al Nahyan Foundation. The funders had no role in study design, data collection and analysis, decision to publish, or preparation of the manuscript. AAA has no conflict of interest to disclose.

\section{Bibliography}

1. Sumit Gupta., et al. "International Trends in the Incidence of Cancer Among Adolescents and Young Adults". JNCI: Journal of the National Cancer Institute (2020).

2. Fidler MM., et al. "Cancer incidence and mortality among young adults aged 20-39 years worldwide in 2012: a population-based study". Lancet Oncology 18.12 (2007): 1579-1589.

3. Al-Shamsi HO and Alrawi S. "Breast cancer screening in the United Arab Emirates: is it time to call for a screening at an earlier age?" Journal of Cancer Prevention and Current Research 9.3 (2018): 123-126.

4. Radwan H., et al. "The epidemiology of cancer in the United Arab Emirates: A systematic review". Medicine (Baltimore) 97.50 (2018).

5. Salim N., et al. "An overview of breast cancer epidemiology, incidence and trends from 2008-2016 in Dubai Hospital, Dubai Health Authority, Hospital-Based Cancer Registry". Journal of Palliative Care and Medicine 8.6 (2018).

6. Howlader N., et al. "SEER Cancer Statistics Review, 19752016". National Cancer Institute. Bethesda, MD.

7. Siegel RL., et al. "Colorectal cancer statistics, 2020". CA: A Cancer Journal for Clinicians (2020).

8. Siegel RL., et al. "Colorectal cancer incidence patterns in the United States, 1974-2013". JNCI: Journal of the National Cancer Institute 109.8 (2017).

9. Welch HG and Robertson DJ. "Colorectal cancer on the decline-why screening can't explain it all”. The New England Journal of Medicine 374.17 (2016): 1605-1607. 
10. Mauri G., et al. "Early-onset colorectal cancer in young individuals". Molecular Oncology 13.2 (2019): 109-131.

11. Bleyer WA., et al. "Cancer in adolescents and young adults" (2017): 127-128.

12. Tricoli JV., et al. "Biologic and clinical characteristics of adolescent and young adult cancers: acute lymphoblastic leukemia, colorectal cancer, breast cancer, melanoma, and sarcoma". Cancer 122.7 (2016): 1017-1028.

13. Chen FW., et al. "Advanced-Stage Colorectal Cancer in Persons Younger Than 50 Years Not Associated With Longer Duration of Symptoms or Time to Diagnosis". Clinical Gastroenterology and Hepatology 15.5 (2017): 728-737.e3.

14. Meester RG., et al. "Trends in incidence and stage at diagnosis of colorectal cancer in adults aged 40 through 49 years, 19752015". JAMA 321.19 (2019): 1933-1934.

15. Dozois EJ., et al. "Young-onset colorectal cancer in patients with no known genetic predisposition: can we increase early recognition and improve outcome?". Medicine 87.5 (2008): 259.

16. Willauer AN., et al. "Clinical and molecular characterization of early-onset colorectal cancer". Cancer 125.12 (2019): 20022010.

17. Fayadh MH. "Colorectal Cancer in Abu Dhabi, UAE - Initial Data 2014-2016". Colorectal Cancer 2 (2016): 3.

18. Makki HF and Salem AS. "Colorectal Cancer in UAE, Implications on the Screening Program 2018". Open Access Journal of Oncology and Medicine 2.2 (2018).

19. Lin JS., et al. "Screening for Colorectal Cancer: A Systematic Review for the US Preventive Services Task Force: Evidence Synthesis No. 135". AHRQ publication 1405203-EF-1. Rockville, MD: Agency for Healthcare Research and Quality (2016).

20. American Cancer Society. Colorectal Cancer Facts and Figures. 2017-2019 (2017).

21. Chyke Doubeni. Screening for colorectal cancer: Strategies in patients at average risk, Uptodate.com.

22. Stoffel EM., et al. "Germline genetic features of young individuals with colorectal cancer". Gastroenterology 154.4 (2018): 897-905.

23. Stoffel EM and Murphy CC. "Epidemiology and mechanisms of the increasing incidence of colon and rectal cancers in young adults". Gastroenterology 158.2 (2020): 341-353.
24. DOH colorectal cancer screening program specifications (2019).

25. Al Abdouli L., et al. "Colorectal cancer risk awareness and screening uptake among adults in the United Arab Emirates". Asian Pacific Journal of Cancer Prevention 19 (2018): 23432349.

\section{Assets from publication with us}

- Prompt Acknowledgement after receiving the article

- Thorough Double blinded peer review

- Rapid Publication

- Issue of Publication Certificate

- High visibility of your Published work

Website: www.actascientific.com/

Submit Article: www.actascientific.com/submission.php

Email us: editor@actascientific.com

Contact us: +919182824667 